\title{
Prognostic factors associated with early gout flare recurrence in patients initiating urate-lowering therapy during an acute gout flare
}

\author{
C. A. Janssen ${ }^{1}$ (D) M. A. H. Oude $\operatorname{Voshaar}^{1}$ - P. M. ten Klooster ${ }^{1} \cdot$ H. E. Vonkeman ${ }^{1,2} \cdot$ M. A. F. J. van de Laar ${ }^{1,2}$
}

Received: 6 February 2019 / Revised: 9 April 2019 / Accepted: 15 April 2019 / Published online: 27 April 2019

(C) The Author(s) 2019

\begin{abstract}
Lowering serum urate levels below the threshold for crystal formation with urate-lowering therapy (ULT) has been associated with a lower risk for gout flare reoccurrences. However, gout patients on ULT still commonly suffer from recurring gout flares. The purpose of this study was to explore prognostic factors associated with gout flare recurrence within the first 3 months, in gout patients starting ULT during an acute gout flare. Post-hoc analysis of trial data on acute gout patients randomized to either gout flare standard of care or anakinra treatment were used, including baseline demographic, laboratory, clinical, and patient-reported variables, as well as 3-month follow-up data on gout flare recurrences. Only patients starting ULT at baseline were included. Using variable selection based on clinical relevance, univariate, and multivariate binary logistic regression analyses were done to examine predictors of gout flare reoccurrence. A total of 75 patients were included in this study, of which 36 (48\%) experienced a gout flare $\leq 3$ months post baseline. The multivariate regression analysis revealed that CRP levels $>30 \mathrm{mg} / \mathrm{L}$ (OR 9.47) and lack of prophylaxis when starting ULT (OR 11.56) were independently associated with gout flare recurrence. Similar results were found for the univariate regression analyses. Our results show that CRP levels $>30 \mathrm{mg} / \mathrm{L}$ and lack of prophylaxis when starting ULT were prognostic factors for early gout flare reoccurrence in patients starting ULT during an acute gout flare.

\section{Key Points}

- Gout flare recurrences were common within the first 3 months after starting urate-lowering therapy in gout patients.

- Intake of prophylaxis when starting ULT had a strong protective effect on gout flare recurrences.

- C-reactive protein level $>30 \mathrm{mg} / \mathrm{L}$ was an additional prognostic factor for early ( $\leq 3 \mathrm{months})$ gout flare reoccurrence in patients starting ULT during an acute gout flare.
\end{abstract}

Keywords Flares · Gout · Prognostic factors · Urate-lowering therapy

\section{Introduction}

Lowering serum urate (SUA) levels below the threshold for monosodium urate crystal formation to the recommended

C. A. Janssen

C.A.Janssen@utwente.nl

1 Department of Psychology, Health and Technology, Arthritis Center Twente, University of Twente, PO BOX 217, 7500

AE Enschede, The Netherlands

2 Department of Rheumatology and Clinical Immunology, Medisch Spectrum Twente, Enschede, The Netherlands
SUA target of $<0.36 \mathrm{mmol} / \mathrm{L}$ with urate-lowering therapy (ULT) has been associated with reduced risk of gout flare recurrences and other adverse outcomes [1, 2]. Therefore, most current gout management guidelines recommend to consider and discuss ULT with every patient, and to initiate ULT for patients with frequent flares, arthropathy, renal impairment, or tophi $[2,3]$. Various guidelines agree that ULT should be initiated early, possibly even upon presentation of an acute gout attack [2-4].

However, research to date shows that even if ULT is instated, gout flare recurrences are relatively common, particularly in the period immediately following initiation $[1,5,6]$. Moreover, previous studies have shown that frequently recurrent gout (i.e., having $\geq 3$ flares per year) is associated with 
work impairment and decreased health-related quality of life $[7,8]$. While preventing recurrent gout flares is considered a priority for improving gout outcomes, not much is currently known about prognostic factors that place patients at increased risk for recurrent gout flares, especially in the period shortly after initiating ULT $[6,9,10]$. In fact, no previous studies have been performed in patients who initiate ULT during an acute gout flare [11-13].

Therefore, in the current study, we investigated the prognostic value of various demographic, clinical or patientreported factors for gout flare recurrence within the first 3 months following ULT initiation, in gout patients starting ULT during an acute gout flare.

\section{Materials and methods}

\section{Data source and patient population}

For this post-hoc analysis, we used data from a multicenter, double-blind, double-dummy, randomized, non-inferiority clinical trial, in which the efficacy of anakinra versus standard of care for the treatment of acute gout flares was studied among crystal-proven gout patients recruited from seven rheumatology clinics in The Netherlands [14]. At baseline, eligible patients with an acute gout flare were randomized to either treatment with anakinra or standard of care, including colchicine, naproxen or prednisolon. In addition, the study protocol stipulated that patients should be initiated on ULT. All patients in the standard of care arm receiving treatment with naproxen or colchicine for their acute gout flare, continued this treatment up for 90 days as flare prophylaxis when initiating ULT. Patients randomized to the standard of care arm and receiving prednisolon, and patients who were randomized to the anakinra arm were provided with medication for their acute gout flare only and were not given prophylaxis with this treatment during initiation of ULT. This was because prophylaxis with corticosteroids or anakinra is currently not recommended in gout guidelines [2]. Patients were followed up for 3 months. More details regarding the study are given elsewhere [14]. This study was approved by a local independent ethics committee and by the institutional review board of each participating center, and was performed in accordance with the declaration of Helsinki and Good Clinical Practice guidelines. All patients provided written informed consent.

For the present study, we excluded patients from the original study who did not initiate ULT at baseline or were lost to follow-up.

\section{Reporting gout flares}

During the 3-month follow-up, patients were instructed to contact their treating rheumatologist or rheumatology nurse when they believed they were experiencing a new gout flare. Evaluator confirmed flares were subsequently documented in the electronic case-report form. Gout flare medication was provided to patients at the discretion of the treating rheumatologist. Beside the evaluator confirmed flares, patients themselves could also report gout flares in their electronic case report form.

\section{Statistical analysis}

For all included patients, baseline characteristics were summarized using mean and standard deviations, number and percentages, or the median and first and third quartile (Q1, Q3), depending on the distribution of the variables. For all statistical analyses, the statistical package SPSS version 22 was used for Windows.

To determine if an association exists between baseline characteristics and the occurrence of a gout flare within the first 3 months following initiation of ULT, a multivariate binary logistic regression model was developed. Potential predictors of flare recurrence were selected from the baseline data, based on their potential clinical relevance, including the levels of patient-reported pain (measured on a 5-point rating scale), body mass index (BMI), sex, levels of creactive protein (CRP) and serum urate (SUA), age, type of gout (intermittent or chronic gout), and receiving prophylaxis during ULT initiation. All potential variables were dichotomized using receiver operating characteristic (ROC) curves with the Youden Index to determine optimal cutoff points.

Preceding the dichotomization of variables and the regression analysis, missing values for the potential predictors (pain $16.0 \%$, BMI $1.3 \%$, CRP $6.7 \%$, SUA $8.0 \%$ ) were replaced by plausible values according to the Multiple Imputation by predictive mean matching method, based on the assumption that the data were missing at random. All baseline variables, as well as ULT type and occurrence of a flare within 3 months post baseline, were used as input for the imputation model [15]. Ten imputed datasets were generated.

Both a weighted univariate and multivariate binary logistic regression analysis was performed with all the potential predictors. The multivariate regression model was done using conditional backward elimination, with $p>0.10$ as the cutoff probability of stepwise removal of variables. As backward elimination on the ten imputed datasets might cause differences in selection of variables for the final multivariate model in each dataset, one analysis was performed in which the ten generated datasets with multiply imputed data were stacked. In that analysis, individual cases were weighted by 0.10 (1/number of datasets of imputation) to correct the standard errors for the artificial inflation of the sample size [16]. The goodness of fit for the model was tested using the Hosmer and Lemeshow test. The model's ability to discriminate between gout flare occurrence (yes/no) was examined by determining 
the area under the ROC curve, whereby an area between 0.70 and $<0.80$ indicated adequate discriminative ability, and between 0.80 and $<0.90$ excellent discriminative ability [17]. For the covariates remaining in the final model at the last step, odds ratios (OR) including corresponding $p$ values and 95\% confidence intervals $(95 \% \mathrm{CI})$ were calculated.

\section{Results}

\section{Patient selection and baseline characteristics}

Of the original 88 included patients, two patients never initiated ULT during the study, eight patients failed to initiate ULT at baseline, and three patients were lost to follow-up, leaving a total of 75 eligible patients who were included in the present analysis. Patient characteristics are provided in Table 1.

The majority of patients $(n=71,94.7 \%)$ initiated ULT with allopurinol, of which $55(77.5 \%)$ had a starting dose of $100 \mathrm{mg}, 13(18.3 \%)$ a starting dose of $300 \mathrm{mg}$, and one (1.4\%) and two (2.8\%) patients a starting dose of $50 \mathrm{mg}$ and $150 \mathrm{mg}$, respectively. Benzbromarone was given as the initial ULT in two patients $(2.7 \%)$ at a starting dose of $50 \mathrm{mg}$, while febuxostat was given to two patients $(2.7 \%)$ of which one patient initiated at a dose of $40 \mathrm{mg}$ and another patient at a dose of $80 \mathrm{mg}$.

Of the total 75 patients included in this analysis, 34 $(45.3 \%)$ received treatment with anakinra at baseline, and 41 (55\%) with standard of care, of which $14(18.7 \%)$ with prednisolon, 16 (21.3\%) with colchicine, and 11 (14.7\%) with naproxen, according to the original study protocol.

Table 1 Baseline patient characteristics

\begin{tabular}{ll}
\hline Characteristics & Total group $(N=75)$ \\
\hline Age, years, mean \pm SD & $61.0 \pm 12.5$ \\
Male sex, $n(\%)$ & $73(97.3)$ \\
BMI, $\mathrm{kg} / \mathrm{m}^{2}$, mean $\pm \mathrm{SD}(N=74)$ & $29.5 \pm 3.9$ \\
$\mathrm{SUA}, \mathrm{mmol} / \mathrm{L}$, mean $\pm \mathrm{SD}(N=69)$ & $0.50 \pm 0.11$ \\
$\mathrm{CRP}, \mathrm{mg} / \mathrm{L}$, median $(\mathrm{Q} 1, \mathrm{Q} 3)(N=70)$ & $14.0(5.0,29.5)$ \\
${\text { Intermittent gout, } n(\%)^{1}}^{1}$ & $59(78.7)$ \\
Pain $^{2}$, mean $\pm \mathrm{SD}(N=63)$ & $3.5 \pm 0.80$ \\
Received prophylaxis, $n(\%)$ & $27(36)$ \\
\hline
\end{tabular}

SD, standard deviation; BMI, body mass index; SUA, serum urate; CRP, C-reactive protein

${ }^{1}$ Compared to patients having chronic gout

${ }^{2}$ Measured on a 5 -point rating scale; $1=$ none, $2=$ mild, $3=$ moderate, $4=\mathrm{a}$ lot, $5=$ extreme

\section{Gout flare recurrence}

In total, 36 (48\%) patients experienced a gout flare, including both evaluator confirmed and patient-reported flares, within the first 3 months after ULT initiation, with a mean (SD) and median $(\mathrm{Q} 1, \mathrm{Q} 3)$ time to the first reported gout flare event of 34.9 (31.8) days and $21.0(12.5,41.8)$ days, respectively. Overall, the mean (SD) number of flares per patient was $0.67(0.81)$ flares. Of the patients that experienced a gout flare, $24(66.7 \%)$ had one flare, 10 (27.8\%) patients had two flares, and two $(5.6 \%)$ patients had three gout flares. The mean (SD) number of flares among the patients having gout flares was $1.39(0.60)$.

The highest proportion of patients experiencing a gout flare recurrence received treatment with anakinra $(n=23,63.9 \%)$, followed by prednisolon $(n=8,22.2 \%)$, naproxen $(n=3$, $8.3 \%)$, and colchicine $(n=2,5.6 \%)$. Interestingly, the first two groups did not receive prophylaxis when starting ULT, while the latter two groups were provided with prophylaxis.

\section{Predictors of flare recurrence}

For all the baseline predictors included in the multivariate regression model, the results of the univariate binary logistic regressions are presented in Table 2.

Results of the multivariate regression analysis showed that the two baseline covariates CRP level and prophylaxis, remained in the model and were independently predictive of gout flare recurrence $(p \leq 0.10)$ (Table 3$)$. The odds for gout flare recurrence were 9.47 times higher for patients with CRP levels above $30 \mathrm{mg} / \mathrm{L}$ compared to patients with CRP levels at or below this value at baseline. In addition, intake of prophylaxis had a strong protective effect (OR, 0.09). The overall goodness to fit of the multivariate model was acceptable $\left(\chi^{2}\right.$ $(7)=2.44 ; p=0.296$ ), and the area under the ROC curve was high, 0.78 , implying the model had adequate discriminative ability.

\section{Discussion}

Our study showed that approximately half of the patients $(48 \%)$ had at least one gout flare recurrence in the first 3 months following ULT initiation during an acute gout flare. Various studies that have previously reported on flare rates, show similar, or higher, percentages of gout patients having flares when on ULT, although differences in study designs and patient populations make comparison difficult $[1,6,11,12$, $18,19]$. Overall, our findings add to the already existing literature reporting on the unfavorable occurrence of gout flares when on ULT.

Furthermore, our results showed that receiving prophylaxis at the moment of ULT initiation strongly protected against 
Table 2 Univariate binary logistic regression analysis of baseline covariates, associated with the occurrence of reported gout flare events $\leq 3$ months post baseline

\begin{tabular}{|c|c|c|c|c|c|}
\hline \multirow[t]{2}{*}{ Baseline variable } & \multicolumn{2}{|c|}{ Flare occurrence, $N(\%)^{1}$} & \multicolumn{3}{|c|}{ Binary logistic regression } \\
\hline & Yes & No & OR & 95\% CI (lower, upper) & $p$ \\
\hline \multicolumn{6}{|l|}{ Pain $^{2}$} \\
\hline$>3$ & $22(56.4)$ & $17(43.6)$ & 2.13 & $0.84,5.35$ & 0.11 \\
\hline$\leq 3$ & $14(38.9)$ & $22(61.1)$ & - & - & - \\
\hline \multicolumn{6}{|l|}{ BMI } \\
\hline$>27 \mathrm{~kg} / \mathrm{m}^{2}$ & $28(50.0)$ & $28(50.0)$ & 1.38 & $0.48,3.93$ & 0.55 \\
\hline$\leq 27 \mathrm{~kg} / \mathrm{m}^{2}$ & $8(42.1)$ & $11(57.9)$ & - & - & - \\
\hline \multicolumn{6}{|l|}{ CRP } \\
\hline$>30 \mathrm{mg} / \mathrm{L}$ & $15(78.9)$ & $4(21.1)$ & 5.90 & $1.72,20.2$ & 0.01 \\
\hline$\leq 30 \mathrm{mg} / \mathrm{L}$ & $22(38.6)$ & $35(61.4)$ & - & - & - \\
\hline \multicolumn{6}{|l|}{ SUA } \\
\hline$>0.39 \mathrm{mmol} / \mathrm{L}$ & $33(50.8)$ & $32(49.2)$ & 2.03 & $0.50,8.13$ & 0.32 \\
\hline$\leq 0.39 \mathrm{mmol} / \mathrm{L}$ & $3(30.0)$ & $7(70.0)$ & - & - & - \\
\hline \multicolumn{6}{|l|}{ Age } \\
\hline$>60$ years & $25(61.0)$ & $16(39.0)$ & 3.27 & $1.26,8.48$ & 0.02 \\
\hline$\leq 60$ years & $11(32.4)$ & $23(67.6)$ & - & - & - \\
\hline \multicolumn{6}{|l|}{ Type of gout } \\
\hline Chronic gout & $8(50.0)$ & $8(50.0)$ & 1.11 & $0.37,3.34$ & 0.86 \\
\hline Intermittent gout & $28(47.5)$ & $31(52.5)$ & - & - & - \\
\hline \multicolumn{6}{|l|}{ Baseline prophylaxis } \\
\hline No & $31(64.6)$ & $17(35.4)$ & 8.02 & $2.57,25.0$ & 0.00 \\
\hline Yes & $5(18.5)$ & $22(81.5)$ & - & - & - \\
\hline \multicolumn{6}{|l|}{ Sex } \\
\hline Female & $1(50.0)$ & $1(50.0)$ & 1.09 & $0.07,18.0$ & 0.95 \\
\hline Male & $35(47.9)$ & $38(52.1)$ & - & - & - \\
\hline
\end{tabular}

gout flare reoccurrence, which further substantiates the importance of prophylaxis when initiating ULT, as is currently recommended by gout management guidelines [2]. The subpopulation of patients that received prophylaxis in our study were provided prophylaxis for 3 months with colchicine or the nonsteroidal anti-inflammatory drug (NSAID) naproxen. This was because we were interested in determining the prognostic value of prophylaxis in preventing gout flares shortly after starting ULT since gout flares are common during this period

Table 3 Multivariate regression model of baseline covariates, associated with the occurrence of reported gout flare events $\leq 3$ months post baseline

\begin{tabular}{lrll}
\hline Baseline variable & \multicolumn{1}{l}{ OR } & $95 \% \mathrm{CI}$ & $p$ \\
\hline $\mathrm{CRP}>30 \mathrm{mg} / \mathrm{L}$ & 9.47 & $2.06-43.51$ & $<0.01$ \\
No prophylaxis & 11.56 & $2.96-45.11$ & $<0.00$ \\
\hline
\end{tabular}

Nagelkerke $R^{2}=0.40$

$\mathrm{OR}$, odds ratio; $\mathrm{CI}$, confidence interval; $\mathrm{CRP}$, C-reactive protein
[1]. We must note, however, that in contemporary gout guidelines prophylaxis with these agents is recommended for the first 6 months when starting ULT [2]. This is supported by data showing that gout flare prevention was more beneficial with intake of 6 months prophylaxis compared to 8 weeks, without the occurrence of more adverse reactions [9]. Future studies would need to evaluate the differences in gout flare risk, but also adverse reactions, between 3 months prophylactic treatment, as applied in this study, compared to the recommended 6 months. Moreover, prophylaxis with agents other than colchicine or NSAIDs may need consideration in the future as well, for example, with interleukin-1 (IL-1) inhibitors. Namely, it has been shown in previous studies that the IL1 inhibitors canakinumab and rilonacept have prophylactic potency in preventing gout flares [19-23]. In the study by Schlesinger et al. 2011, prophylaxis with single doses of canakinumab $\geq 50 \mathrm{mg}$ or four 4 -weekly doses $(50 \mathrm{mg}$, $50 \mathrm{mg}, 25 \mathrm{mg}, 25 \mathrm{mg}$ ) provided a greater relief in gout flare recurrences compared to daily colchicine $0.5 \mathrm{mg}$ given for 16 weeks in gout patients starting ULT with allopurinol [20]. 
In this study, less patients experienced at least one gout flare in the study period when treated with canakinumab (15-18.5\%) versus colchicine $(44 \%)$. The various studies done on rilonacept (once weekly subcutaneous injection of 80 or $160 \mathrm{mg}$ ) also revealed that it was more beneficial in reducing the frequency of gout flare recurrences compared to placebo when on ULT. Here, the proportion of patients experiencing at least one gout flare in 16 weeks ranged from 16 to $26 \%$ for rilonacept and 47 to $56 \%$ for placebo [19, 21-23]. Prophylactic treatment with IL-1 inhibitors may in particular be of relevance in those gout patients who cannot be treated with colchicine or NSAIDs.

Moreover, CRP levels above $30 \mathrm{mg} / \mathrm{L}$ were independently predictive of gout flare recurrence. To our knowledge, limited research is currently available on the possible relationship between CRP levels and developing recurrent gout flares. In previous studies, increased systemic inflammation, including elevated markers as CRP, has been associated with elevated SUA levels and with cardiovascular diseases [24-28]. Therefore, the potential role of CRP in predicting gout flares, and the nature of this possible association, including such underlying factors (e.g., SUA level, presence of comorbidities), may need to be explored in future studies, particularly in other gout populations.

Previous long-term ( $\geq 1$ year) retrospective studies have shown an association between increased SUA levels and an increased risk for gout flare occurrence [1, 11, 12, 18, 29]. Contrarily, results of our study did not reveal such an association and SUA levels to be prognostic for the reoccurrence of a gout flare in the short-term ( $\leq 3$ months). Although the reason for this observation cannot be determined from our study, it might be attributed to the fact that the study population was rather homogenous, consisting primarily of patients having a baseline SUA level $($ mean $=0.50 \mathrm{mmol} / \mathrm{L})$ well above the recommended SUA target value of $0.36 \mathrm{mmol} / \mathrm{L}$. Notably, previous studies that have applied the SUA value of $0.36 \mathrm{mmol} / \mathrm{L}$ as the cutoff value for dichotomization in regression models found an association between SUA level and gout flares, whereas in the present study we used a SUA dichotomization value of $0.39 \mathrm{mmol} / \mathrm{L}$ and found no association [1, $11,12,18]$.

The various clinical and patient-related baseline characteristics considered as potential prognostic factors, as well as the use of data in patients with crystal-proven gout are strengths of this study. However, this study also has some limitations. First, potential predictors such as the presence of comorbidities, medication usage, and diet (e.g., purine-rich foods) were not available for this study, although these have been suggested to be relevant with regard to gout flare reoccurrence [30-32]. This includes the effect of different types of ULT on gout flare risk, which we were not able to include as a predictor in the model due to the variety of ULT and starting doses that were applied, leading to a small number of subjects receiving each ULT. Also, patients' actual adherence to their ULT medication during the course of the study was not measured. Moreover, our study population was limited to patients treated at rheumatology centers, and our results should therefore be confirmed in studies in different populations. Another limitation of this study is that the recently validated definition of gout flares for use in gout clinical research was not used in this study, but gout flares reported by patients and evaluators were used [33]. Finally, as we studied predictors of gout flare recurrence in patients initiating ULT during an acute gout episode, the generalizability of our results to gout patients not on ULT, and starting ULT prior to or following a gout flare, is limited.

In conclusion, we demonstrated that CRP levels $>30 \mathrm{mg} / \mathrm{L}$ and lack of prophylaxis were independent, prognostic factors for early ( $\leq 3$ months) gout flare reoccurrence in gout patients initiating ULT during an acute gout flare.

Funding information This work was supported by a grant from The Netherlands Organisation for Health Research and Development (ZonMw) under its program Rational Pharmacotherapy [836031015]. Swedish Orphan Biovitrum AB (publ) provided grants for the conduct of the original randomized controlled trial.

\section{Compliance with ethical standards}

Conflict of interest Unrestricted educational grants and non-financial support from Swedish Orphan Biovitrum AB during the conduct of the study, and an unrestricted educational grant from Grünenthal B.V. outside the submitted work, were declared by C.A. Janssen, M.A.H. Oude Voshaar, and M.A.F.J. van de Laar. Also, M.A.F.J van de Laar reports consultancy work/speaking for Grünenthal B.V. and Swedish Orphan Biovitrum AB. The other authors declare to have no conflicts of interest.

Ethical standards The study was approved by an independent ethics committee (METC Twente, Enschede, the Netherlands), and by the institutional review board of each participating center. It has therefore been performed in accordance with ethical standard laid down in the 1964 Declaration of Helsinki and its later amendments, and the Dutch Medical Research Involving Human Subjects Act. All patients provided written informed consent prior to study initiation.

Open Access This article is distributed under the terms of the Creative Commons Attribution 4.0 International License (http:// creativecommons.org/licenses/by/4.0/), which permits unrestricted use, distribution, and reproduction in any medium, provided you give appropriate credit to the original author(s) and the source, provide a link to the Creative Commons license, and indicate if changes were made.

\section{References}

1. Sarawate CA, Patel PA, Schumacher HR, Yang W, Brewer KK, Bakst AW (2006) Serum urate levels and gout flares analysis from managed care data. J Clin Rheumatol 12:61-65. https://doi.org/10. 1097/01.rhu.0000209882.50228.9f

2. Richette P, Doherty M, Pascual E, Barskova V, Becce F, CastañedaSanabria J, Coyfish M, Guillo S, Jansen TL, Janssens H, Lioté F, 
Mallen C, Nuki G, Perez-Ruiz F, Pimentao J, Punzi L, Pywell T, So A, Tausche AK, Uhlig T, Zavada J, Zhang W, Tubach F, Bardin T (2017) 2016 updated EULAR evidence-based recommendations for the management of gout. Ann Rheum Dis 76:29-42. https:// doi.org/10.1136/annrheumdis-2016-209707

3. Hui M, Carr A, Cameron S, Davenport G, Doherty M, Forrester H, Jenkins W, Jordan KM, Mallen CD, McDonald T, Nuki G, Pywell A, Zhang W, Roddy E, British Society for Rheumatology Standards, Audit and Guidelines Working Group (2017) The British Society For Rheumatology guideline for the management of gout. Rheumatology 56:e1-e20. https://doi.org/10.1093/ rheumatology/kex 150

4. Khanna D, Fitzgerald JD, Khanna PP, Bae S, Singh MK, Neogi T, Pillinger MH, Merill J, Lee S, Prakash S, Kaldas M, Gogia M, Perez-Ruiz F, Taylor W, Lioté F, Choi H, Singh JA, Dalbeth N, Kaplan S, Niyyar V, Jones D, Yarows SA, Roessler B, Kerr G, King C, Levy G, Furst DE, Edwards NL, Mandell B, Schumacher HR, Robbins M, Wenger N, Terkeltaub R, American College of Rheumatology (2012) 2012 American college of rheumatology guidelines for management of gout. Part 1: systematic nonpharmacologic and pharmacologic therapeutic approaches to hyperuricemia. Arthritis Care Res 64:1431-1446. https://doi.org/ 10.1002/acr.21772

5. Doherty M, Jansen TL, Nuki G, Pascual E, Perez-Ruiz F, Punzi L, So AK, Bardin T (2012) Gout: why is this curable disease so seldom cured? Ann Rheum Dis 71:1765-1770. https://doi.org/10. 1136/annrheumdis-2012-201687

6. Becker MA, MacDonald PA, Hunt BJ et al (2008) Determinants of the clinical outcomes of gout during the first year of urate-lowering therapy. Nucleosides Nucleotides Nucleic Acids 27:585-591. https://doi.org/10.1080/15257770802136032

7. Khanna PP, Nuki G, Bardin T, Tausche AK, Forsythe A, Goren A, Vietri J, Khanna D (2012) Tophi and frequent gout flares are associated with impairments to quality of life, productivity, and increased healthcare resource use: results from a cross-sectional survey. Health Qual Life Outcomes 10:1-11. https://doi.org/10.1186/ 1477-7525-10-117

8. Edwards NL, Sundy JS, Forsythe A, Blume S, Pan F, Becker MA (2011) Work productivity loss due to flares in patients with chronic gout refractory to conventional therapy. J Med Econ 14:10-15. https://doi.org/10.3111/13696998.2010.540874

9. Wortmann RL, MacDonald PA, Hunt B, Jackson RL (2010) Effect of prophylaxis on gout flares after the initiation of urate-lowering therapy: analysis of data from three phase III trials. Clin Ther 32: 2386-2397. https://doi.org/10.1016/j.clinthera.2011.01.008

10. Dalbeth N, Choi HK, Terkeltaub R (2017) Gout: a roadmap to approaches for improving global outcomes. Arthritis Rheumatol 69:22-34. https://doi.org/10.1002/art.39799

11. Rashid N, Levy GD, Wu Y-L, Zheng C, Koblick R, Cheetham TC (2015) Patient and clinical characteristics associated with gout flares in an integrated healthcare system. Rheumatol Int 35:17991807. https://doi.org/10.1007/s00296-015-3284-3

12. Halpern R, Fuldeore MJ, Mody RR, Patel PA, Mikuls TR (2009) The effect of serum urate on gout flares and their associated costs: an administrative claims analysis. J Clin Rheumatol 15:3-7. https:// doi.org/10.1097/RHU.0b013e3181945d2c

13. Wu E, Patel P, Mody R et al (2009) Frequency, risk, and cost of gout-related episodes among the elderly: does serum uric acid level matter? J Rheumatol 36:1032-1040. https://doi.org/10.3899/ jrheum.080487

14. Janssen CA, Oude Voshaar MAH, Vonkeman HE, Jansen TLTA, Janssen M, Kok MR, Radovits B, van Durme C, Baan H, van de
Laar MAFJ (2019) Anakinra for the treatment of acute gout flares: a randomized, double-blind, placebo-controlled, active-comparator, non-inferiority trial. Rheumatology. https://doi.org/10.1093/ rheumatology/key 402

15. Sterne JAC, White IR, Carlin JB, Spratt M, Royston P, Kenward MG, Wood AM, Carpenter JR (2009) Multiple imputation for missing data in epidemiological and clinical research: potential and pitfalls. BMJ 338:b2393. https://doi.org/10.1136/bmj.b2393

16. Wood AM, White IR, Royston P (2008) How should variable selection be performed with multiply imputed data? Stat Med 27: 3227-3246. https://doi.org/10.1002/sim.3177

17. Hosmer DW, Lemeshow S (2000) Applied logistic regression, 2nd edn. Wiley, New York

18. Annemans L, Spaepen E, Gaskin M, Bonnemaire M, Malier V, Gilbert T, Nuki G (2008) Gout in the UK and Germany: prevalence, comorbidities and management in general practice 2000-2005. Ann Rheum Dis 67:960-966. https://doi.org/10.1136/ard.2007.076232

19. Sundy JS, Schumacher HR, Kivitz A, Weinstein SP, Wu R, KingDavis S, Evans RR (2014) Rilonacept for gout flare prevention in patients receiving uric acid-lowering therapy: results of RESURGE, a phase III, international safety study. J Rheumatol 41:1703-1711. https://doi.org/10.3899/jrheum.131226

20. Schlesinger N, Mysler E, Lin H-Y, de Meulemeester M, Rovensky J, Arulmani U, Balfour A, Krammer G, Sallstig P, So A (2011) Canakinumab reduces the risk of acute gouty arthritis flares during initiation of allopurinol treatment: results of a double-blind, randomised study. Ann Rheum Dis 70:1264-1271. https://doi.org/ 10.1136/ard.2010.144063

21. Mitha E, Ralph Schumacher H, Fouche L et al (2013) Rilonacept for gout flare prevention during initiation of uric acid-lowering therapy: results from the PRESURGE-2 international, phase 3, randomized, placebo-controlled trial. Rheumatol (United Kingdom) 52:1285-1292. https://doi.org/10.1093/rheumatology/ket114

22. Schumacher HR, Evans RR, Saag KG et al (2012) Rilonacept (interleukin-1 trap) for prevention of gout flares during initiation of uric acid-lowering therapy: results from a phase III randomized, double-blind, placebo-controlled, confirmatory efficacy study. Arthritis Care Res 64:1462-1470. https://doi.org/10.1002/acr. 21690

23. Schumacher HR, Sundy JS, Terkeltaub R et al (2012) Rilonacept (interleukin-1 trap) in the prevention of acute gout flares during initiation of urate-lowering therapy: results of a phase II randomized, double-blind, placebo-controlled trial. Arthritis Rheum 64: 876-884. https://doi.org/10.1002/art.33412

24. Kang D, Park S, Lee I, Johnson R (2005) Uric acid-induced Creactive protein expression: implication on cell proliferation and nitric oxide production of human vascular cells. J Am Soc Nephrol 16:3553-3562. https://doi.org/10.1681/asn.2005050572

25. Grainger R, McLaughlin RJ, Harrison AA, Harper JL (2012) Hyperuricaemia elevates circulating CCL2 levels and primes monocyte trafficking in subjects with inter-critical gout. Rheumatol (United Kingdom) 52:1018-1021. https://doi.org/10. 1093/rheumatology/kes326

26. Keenan T, Blaha M, Nasir K et al (2012) Relation of uric acid to serum levels of high-sensitivity C-reactive protein, triglycerides, and high-density lipoprotein cholesterol and to hepatic steatosis. Am J Cardiol 110:1787-1792. https://doi.org/10.1016/j.amjcard. 2012.08.012

27. Mason JC, Libby P (2015) Cardiovascular disease in patients with chronic inflammation: mechanisms underlying premature cardiovascular events in rheumatologic conditions. Eur Heart J 36:482489. https://doi.org/10.1093/eurheartj/ehu403 
28. Kaplan RC, Frishman WH (2001) Systemic inflammation as a cardiovascular disease risk factor and as a potential target for drug therapy. Heart Dis 3:326-332. https://doi.org/10.1097/00132580200109000-00009

29. Shoji A, Yamanaka H, Kamatani N (2004) A retrospective study of the relationship between serum urate level and recurrent attacks of gouty arthritis: evidence for reduction of recurrent gouty arthritis with antihyperuricemic therapy. Arthritis Rheum 51:321-325. https://doi.org/10.1002/art.20405

30. Hunter D, York M, Chaisson C, Woods R, Niu J, Zhang Y (2006) Recent diuretic use and the risk of recurrent gout attacks: the online case-crossover gout study. J Rheumatol 33:1341-1345

31. Primatesta P, Plana E, Rothenbacher D (2011) Gout treatment and comorbidities: a retrospective cohort study in a large US managed care population. BMC Musculoskelet Disord 12:103. https://doi. org/10.1186/1471-2474-12-103

32. Zhang Y, Chen C, Choi H, Chaisson C, Hunter D, Niu J, Neogi T (2012) Purine-rich foods intake and recurrent gout attacks. Ann Rheum Dis 71:1448-1453. https://doi.org/10.1136/annrheumdis2011-201215

33. Gaffo A, Dalbeth N, Saag K et al (2018) Brief report: validation of a definition of flare in patients with established gout. Arthritis Rheumatol 70:462-467. https://doi.org/10.1002/art.40381

Publisher's note Springer Nature remains neutral with regard to jurisdictional claims in published maps and institutional affiliations. 